\title{
Compare various Circuits Area Reduction using Genetic Algorithm and Hybrid Partitioning Algorithm
}

\author{
${ }^{1}$ P Srikanth, ${ }^{2}$ SrijaM, ${ }^{3}$ Chakravarthy S, ${ }^{4}$ G.V.Rao, ${ }^{5}$ GARaju \\ $1,2,3,4$ Department of CSE, Godavari Institute of Engineering \& Technology, Rajahmundry, A.P, India
}

\begin{abstract}
The Floor-planning will be the design flow of VLSI chip methodology. The physical design phases include virtual configuration realizations iterated to their effectiveness. For this persistence, the CAD algorithm provides an assortment of results contingent upon the requirements and determinations of designer. The utilization of tools of EDA assistance for imagining of impacts of the design methodologies on circuit execution and measurements of floor region possessed toward the design. It also utilizes a genetic algorithm (GA). The genetic algorithm has been executed and tested on popular standard issues. The evaluation outcomes indicate that GA might rapidly produce optimal results for all tested benchmark issues. The hybrid algorithm on circuit design is impact for applying the KL, FM (move based separation) algorithms to circuits and optimizing the cells of circuit utilizing HGA (hybrid genetic algorithm)is examined. The outcomes recommend this methodology on circuit gives extent to unify the physical design phases also enhance the physical design's are a factor of a procedure.
\end{abstract}

Keywords: EDA tools, GA, HGA, Floor planning, CAD algorithm

\section{INTRODUCTION}

The floor planning chooses how to organize modules on chip. The partitioning may be the initial stage towards the manufacture of chip from a design. The Floor-plans might a chance to be isolated under two types, the non-slicing structure and the slicing structure. A slicing structure could be indicated by a binary tree whose leaves indicate internal hubs and modules define the cut lines of vertical or horizontal [1] The Non-slicing floor plan has separate strategies such as $\mathrm{B}^{*}$ tree, sequence pair, o-tree, and BSG (bounded slicing grid). The sequence pairs could be utilized to floor plan difficult rectangular pieces by simulated strengthening. To control non-slicing floor-plans, this manuscript recommends an iterative optimized schema that utilizes GA for local examine on every iteration. Inheriting from the specific qualities of the requested $\mathrm{B}^{*}$ - tree has a significant benefits compared for different representations. The $\mathrm{B}^{*}$ tree may be precise adaptable exceptionally and not difficult to usage [2]. It doesn't need should build demand graphs to zone cosset assessment.
Table 1: Chip Performance, Fabrication, and Area for diverse designs

\begin{tabular}{|c|c|c|c|c|}
\hline $\begin{array}{c}\text { Paramete } \\
\mathrm{r}\end{array}$ & \multicolumn{4}{|c|}{ Style } \\
\hline & $\begin{array}{c}\text { Standard } \\
\text { cell }\end{array}$ & $\begin{array}{c}\text { FPG } \\
\text { A }\end{array}$ & $\begin{array}{c}\text { Full } \\
\text { custom }\end{array}$ & Gate array \\
\hline $\begin{array}{c}\text { Performanc } \\
\mathrm{e}\end{array}$ & $\begin{array}{c}\text { High } \\
\mathrm{t}\end{array}$ & Low & High & moderate \\
& $\begin{array}{c}\text { o } \\
\text { moderate }\end{array}$ & & & \\
\hline Area & $\begin{array}{c}\text { Compact } \\
\text { to } \\
\text { Moderat } \\
\mathrm{e}\end{array}$ & Large & $\begin{array}{c}\text { Compac } \\
\mathrm{t}\end{array}$ & Moderate \\
& $\begin{array}{c}\text { All layers } \\
\text { Fabricate }\end{array}$ & $\begin{array}{c}\text { No } \\
\text { layers }\end{array}$ & $\begin{array}{c}\text { All } \\
\text { layers }\end{array}$ & $\begin{array}{c}\text { Routin } \\
\mathrm{g} \\
\text { layers } \\
\text { only }\end{array}$ \\
\hline
\end{tabular}

Table 1 portrays the substance of different plan style to factor under attention. The examination in table 1, it might give conservative layouts to high execution outlines and the FPGA may be totally manufacture and obliges no client particular creation phases[3] The phase of separation, however entitles as a differentiate step; it will be approved best through the placement phase.

\section{PROBLEM FORMULATION}

In hybrid partitioning algorithm, the circuit will be pictured as a graph. A hyper graph $G=(V, E)$ of circuit is deliberated. The $E=\left\{e_{1}, e_{2}, \ldots, e_{m}\right\}$ represent as set of hyper edges and $V=\left\{v_{1}, v_{2}, \ldots, v_{n}\right\}$ represent as set of vertices of hyper graph. Every vertex signifies a hyper edge and a component joining vertices; when component equivalent to these vertices is to be associated [4] [5] Therefore every hyper edge will be subset of vertex set. In other terms, every net will be signified by a hyper edge.

The $\mathrm{V}$ is divided into where and. The partition will be also mentioned to as cut in graph. Partitioning cost is also known as size of cut that will the number of Hyper edges crossing the cut. The function of cost minimization is as represents in equation 1 below: 


$$
\begin{aligned}
& \mathrm{C}=k \\
& k \\
& j=1 \\
& I i, j \quad i \neq \\
& j \ldots . . . . .(1)
\end{aligned}
$$

Here, $=$ cost of edge, $=$ cost of cut, and are the vertices of an edge. As the problem includes circuit's [6] bi-partitioning, condition of equality should be fulfilled in eq (2):

$k$

$i=0$

$[m i] \neq k$

$[n j]$

Here, are the nodes in 2 partitions. This generates the first input netlist from the circuit and estimates the calculation of gain. The complete process on numerous procedures is deliberate previously selecting move based FM algorithm and KL algorithm. The procedure pseudo codes for FM and KL are executed for the hybrid genetic algorithm and move based partitioning with min cut technique is executed for optimization [7].

2.1 Poems Algorithm: POEMS algorithm works in iteration. The goal of the POEMS method is to discover the better adjustment of the model for utilization of the GA that helps as an adjustment optimizer. The function of fitness of every action sequence is characterized as a prototype fitness result following continuously changed toward the specific sequence assessed the whole program. A model is the first result made and progressed through the POEMS methodology[9 Its production is a significant phase, since the first position profoundly impacts the space searched.

Best-fit heuristic will be a usual term for the greedy rectangle packing methodology. The standard will be to choose better fitted segment for every hole in last location. Modules and holes are kept in an algorithm [8] repeat still the module queue may be nil. Subsequently every module placement, the entire list will be updated.

Each movement in the POEMS methodology speaks a specific adjustment of the model. Each activity has a Boolean banner that empowers or deactivates the movement. Whether the activity may be deactivated, it prepares nothing to input tree. Unique movements are combined together to successions that are enhanced through GA. This GA method utilized 6 activities- mirror action, flip action, exchange node action, rotate action, hang node action, and exchange value action. Pattern is indicated in figure 1.

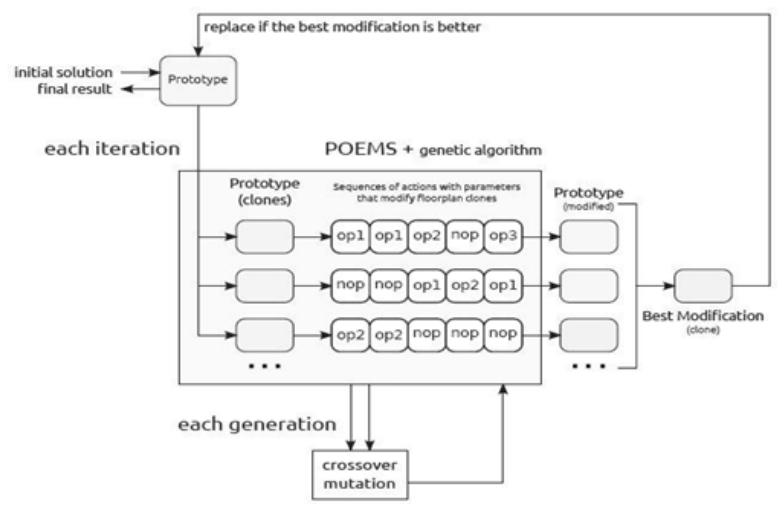

Figure 1: Complete program diagram

GA (Genetic Algorithm): The GA is a technique for resolving constrained optimization and [9] unconstrained optimization issues. In every stage, GA chooses people at casual from with the present population to be guardians and utilization them to prepare the kids for following generation. Again progressive generations, population evolves towards by an optimal result. Here every single person in population of the GA portrayed an arrangement of actions that whether connected to the model floor-plan, makes an alternate floor-plan.

The circuit may be changed as a net list then methodologies are applied. At first hubs are divided utilizing KL algorithms and then min cut algorithm combined with for GA may be utilized as HGA to diminish the region factor of circuit during gate level.

The optimization will be attained utilizing the GA methodology. Further GA may be infused with the HGA and min cut algorithm execution on test circuit utilizing KL methods and FM methods. Min cut algorithm characterizes GA fitness function, consequently making it HGA.

\section{EXPERIMENTAL RESULTS}

The evaluation used GSRC/MCNC benchmarks for VLSI floor-planning issue. It compared with Differential evolution (DE) algorithms and Simulated Annealing (SA). The outcomes attained

Table 2: area comparisons

\begin{tabular}{|c|c|c|c|c|}
\hline Circuits & $\begin{array}{c}\text { No. of } \\
\text { Modul }\end{array}$ & $\begin{array}{c}\text { DE Area } \\
(\mathrm{mm} 2)\end{array}$ & $\begin{array}{c}\text { SA } \\
\text { A }\end{array}$ & $\begin{array}{c}\text { GA } \\
\text { Are }\end{array}$ \\
\hline Hp & 9 & 9.293 & 9.57 & 8.83 \\
\hline ami49 & 49 & 36.22 & 43.34 & 35.45 \\
\hline Xerox & 10 & 19.19 & 20.47 & 19.35 \\
\hline ami33 & 33 & 1.22 & 1.36 & 1.17 \\
\hline
\end{tabular}

In this paper is enhanced comparison to further methods. The evaluation outcomes for area estimation are represented inTable.2.

\subsection{GSRC benchmark}

GSRC is used to select and tested the 2 benchmarks, and they are $\mathrm{n} 10, \mathrm{n} 100$ shown the below table 3 . 
Table 3: The outcomes are depicted

\begin{tabular}{|l|c|c|c|c|c|c|}
\hline Test & $\mathrm{I}$ & $\mathrm{N}$ & $\mathrm{G}$ & $\mathrm{S}$ & Unused area & Unused area \\
\hline $\mathrm{n} 100$ & 10 & 3 & 10 & 50 & 0.036 & 0.036 \\
$\mathrm{n} 100$ & 100 & 3 & 10 & 50 & 0.025 & 0.025 \\
\hline $\mathrm{n} 10$ & 10 & 3 & 10 & 50 & 0.017 & 0.017 \\
$\mathrm{n} 10$ & 100 & 3 & 10 & 50 & 0.009 & 0.009 \\
\hline
\end{tabular}

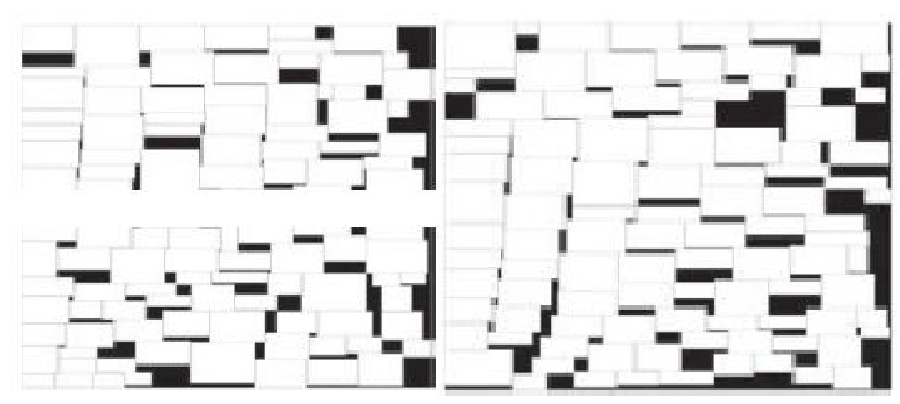

Figure 2: (a): n100 (17\% Dead when $\mathrm{I}=10$ )

(b) $\mathrm{n} 100(12.4 \%$ Dead when $\mathrm{I}=100)$

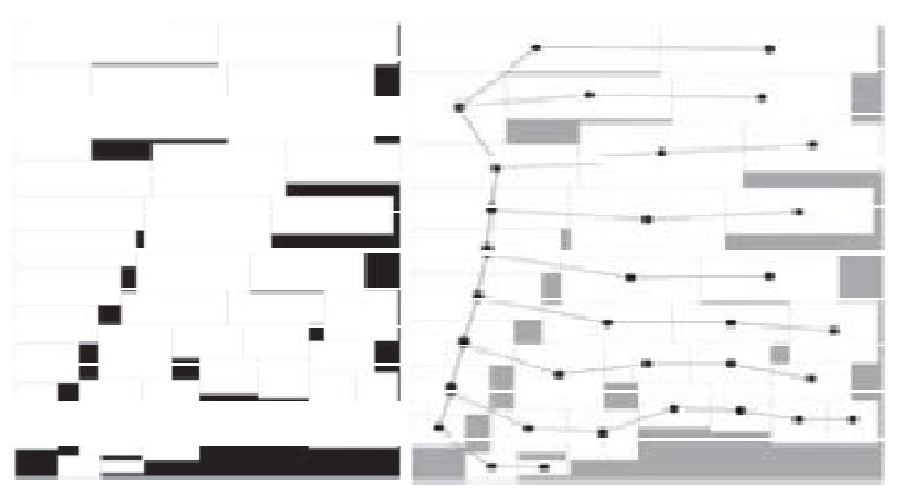

Figure 3: (c) Ami33 (12.3\% dead Fig .(d) B* tree of the placement

\subsection{MCNC benchmark:}

The MCNC is used to select and tested the 5 benchmarks, and they are ami33, ami49, apte, hp, Xerox. The outcomes are represented in the below table. The procedure execution is similar to the GSRC as shown in figures $2 \& 3$. The outcome of this MCNC displays that as number of iteration enhances the improved optimizations attained by reducing the unused area.
Table .4: Results of MCNC benchmark

\begin{tabular}{|l|c|c|c|c|c|c|}
\hline \multicolumn{1}{|c|}{ Test } & I & G & N & S & $\begin{array}{c}\text { Used } \\
\text { area }\end{array}$ & $\begin{array}{c}\text { Unused area } \\
\left(\mathrm{mm}^{2}\right)\end{array}$ \\
\hline Ami49 & 10 & 10 & 3 & 50 & 35.44 & 7.4 \\
Ami49 & 100 & 10 & 3 & 50 & 35.44 & 5.65 \\
\hline Hp & 10 & 10 & 3 & 50 & 8.83 & 0.883 \\
Hp & 100 & 10 & 3 & 50 & 8.83 & 0.553 \\
\hline Ami33 & 10 & 10 & 3 & 50 & 1.16 & 0.162 \\
Ami33 & 100 & 10 & 3 & 50 & 1.16 & 0.082 \\
\hline Xerox & 10 & 10 & 3 & 50 & 19.35 & 1.78 \\
Xerox & 100 & 10 & 3 & 50 & 19.35 & 1.57 \\
\hline
\end{tabular}

All the comparison results shown the table $4 \mathrm{MCNC}$ bench mark. Is HGA is utilized to mine the optimized region for the provided circuit. The dissimilar optimizations attained for C1908, C3540circuits are shown in below graph. The outcomes are displayed in below figure 4 .

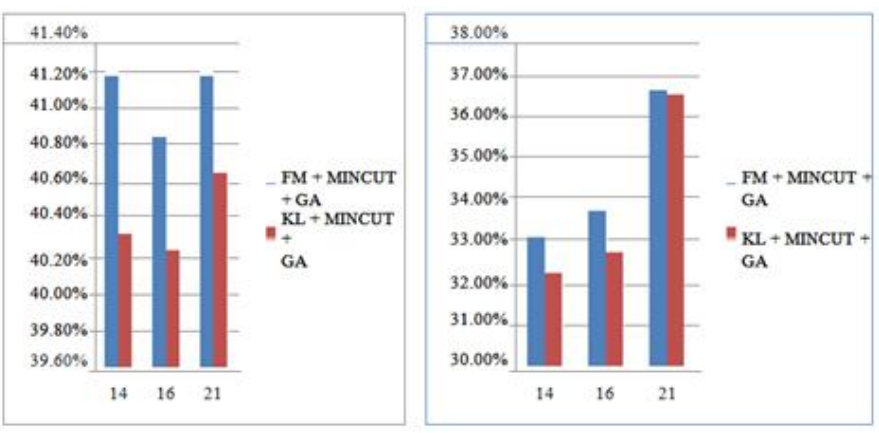

Figure 4: Comparison of C3540, C1908 circuit to show the percentage area reduction

\section{CONCLUSION}

This manuscript contracts with benchmark applications toward gate level, zone optimization utilizing evaluation built genetic algorithm method. The HGA connected to the separating and set hubs and unifies the 2 outline stages of floor arranging and separating. The outcomes recommend that an entire region optimization to a level for $40 \%$ between differentiate small sized and middle sized circuits of benchmark. It emphasizes on versatility of select the stream of procedures relay upon circuit's application complexity.

The GA algorithm will be executed utilizing the java and tested on open benchmark information accessible on the online. The examinations indicate that the proposed algorithm will be aggressive in quality, and even somewhat enhanced than all the further tested methods. Consequently a result for 2D rectangle packing issue will be designed, executed and tested. Presently additional improvement of the algorithm might incorporate rectilinear modules, delicate modules. Additional probability is to estimate wire length among blocks. In that situation estimation of the fitness will a chance to be modified. 


\section{REFERENCES}

[1] P.-N. Guo, C.-K. Cheng, and T. Yoshimura, "An O-Tree Representation of Non- Slicing Floor planned Its Applications," Proc.DAC, pp. 268-273, 1999.

[2] Y. C. Chang, Y. W. Chang, G. M. Wu, andS. W. Wu,"B*Trees: A new representation for non slicing floor plans," Design Automation Conference, pp. 458-463. 2000

[3] D.E. Goldberg, "Genetic Algorithms in Search, Optimization and Machine learning", Pearson Education, ISBN: 13: 9780201157673,2004

[4] M. Tang, and X. Yao, "A Memetic Algorithm for VLSI Floor planning", IEEE Transactions on Systems, Man, and Cybernetics, Part B, vol. 37, pp. 62-69,2007. https://doi.org/10.1109/TSMCB.2006.883268

[5] Christopher J. Augeri Hesham H. Ali, "New GraphBased Algorithms for Partitioning VLSI Circuits", In proceedings of International symposiumon Circuits and Systems,ISCAS, 2004, vol-4,pp:521-524,2004

[6] B.W. Kerhinghan, S. Lin, "An efficient heuristic procedure for partitioning graphs", Bell System Tech. Journal, Vol. 49, pp. 291-307,1970 https://doi.org/10.1002/j.1538-7305.1970.tb01770.x

[7] DavidA.PapaandIgorL.Markov,"Hypergraphpartitioningan dclustering",InApproximation AlgorithmsandMetaheuristic s,CRC press,ISBN:61-1-61-19,2007.

[8] Naveed Sherwani, "Algorithms for VLSI Physical Design and Automation", third edition, Springer (India)Private Limited, New Delhi, ISBN: 0792383931,2005

[9] Pinaki Mazumder, "Genetic Algorithms for VLSI Design, Layout and Test Automation", Addison-Wesley, 